\title{
ASPECTOS ESTÉTICOS EM DEMONSTRAÇÕES DE BONAVENTURA CAVALIERI
}

\section{AESTHETICS ASPECTS IN BONAVENTURA CAVALIERI'S PROOFS}

\author{
Ricardo Scucuglia Rodrigues da Silva ${ }^{1}$, Inocêncio Fernandes Balieiro Filho², Luis \\ Augusto Schimidt Totti ${ }^{3}$, Giovana Aparecida Bertolucci ${ }^{4}$
}

\begin{abstract}
RESUMO
A demonstração matemática evoca questões complexas de natureza lógico-epistemológica. Neste artigo exploramos episódios na história do cálculo infinitesimal, em particular sobre Bonaventura Cavalieri (1598-1647), visando destacar aspectos da demonstração matemática. Inicialmente, apresentamos perspectivas sobre estética e demonstração matemática visando identificar elementos qualitativos na produção de conhecimentos matemáticos (formais). Em seguida, por meio de pesquisa bibliográfica, exploramos o uso de notações/simbologias no desenvolvimento do Princípio de Cavalieri e em resultados envolvendo integração. Enfatizamos aspectos sobre linguagem/notações e procedimentos na enunciação e demonstração de alguns teoremas/proposições de Cavalieri. Destacamos o uso dos símbolos/notações o.l. (omnes linae), o.q. (omnes quadrata) e o.c. (omnes cubi), que significam respectivamente todas as retas ou soma de todas as retas, todos os quadrados e todos os cubos. A noção omnes nesse contexto está relacionada ao conceito de integração definida do Cálculo contemporâneo. Finalmente, mencionamos nuances sobre como Leibniz (1646-1716), ao criar o símbolo de integral a partir da noção de omnes, reorganizou aspectos estéticos da demonstração de Cavalieri e desenvolveu novos resultados sobre integrais.
\end{abstract}

Palavras-chave: História da Matemática. Cálculo Infinitesimal. Demonstração Matemática.

${ }^{1}$ Doutor em Education Studies pela The University of Western Ontario (UWO). Professor Assistente Doutor do Departamento de Educação da Universidade Estadual Paulista (UNESP), Campus de São José do Rio Preto, São Paulo, Brasil. Endereço para correspondência: Rua Rua Cristóvão Colombo, 2265 Jardim Nazareth - São José do Rio Preto/SP - CEP 15054-000. E-mail: ricardo.scucuglia@unesp.br.

(i) ORCID iD: https://orcid.org/0000-0002-5810-2259.

2 Doutor em Educação Matemática pela Universidade Estadual Paulista (UNESP); Professor Assistente Doutor do Departamento de Matemática da Universidade Estadual Paulista (UNESP), Campus de Ilha Solteira, São Paulo, Brasil. Endereço para correspondência: Alameda Rio de Janeiro, 266, Centro, Ilha Solteira, São Paulo, Brasil, CEP 15.385-000. E-mail: inocencio.balieiro@unesp.br

(iD) ORCID iD: https://orcid.org/0000-0003-4012-959X.

3 Doutor em Letras Clássicas pela Universidade de São Paulo (USP). Professor Assistente Doutor do Departamento de Estudos Linguísticos e Literários da Universidade Estadual Paulista (UNESP), Campus de São José do Rio Preto, São Paulo, Brasil. Endereço para correspondência: Rua Rua Cristóvão Colombo, 2265 - Jardim Nazareth - São José do Rio Preto/SP - CEP 15054-000. E-mail: luis.totti@unesp.br

(D) ORCID iD: https://orcid.org/0000-0002-8874-4770

${ }^{4}$ Mestra em Ensino e Processos Formativos pela UNESP (interunidades FCAV, FEIS, IBILCE), obtida em 2020. Professora de Matemática efetiva na SME de Novo Horizonte, São Paulo, Brasil. Endereço para correspondência: Rua Elmezindo Pinto da Silva, $\mathrm{n}^{\circ}$ 162, bairro Jardim Almice - Novo Horizonte, SP, Brasil. CEP: 14960-000. Email: giovana.aparecida@unesp.br

ORCID iD: https://orcid.org/0000-0001-6942-4993. 


\begin{abstract}
Mathematical demonstration evokes complex issues of logical-epistemological nature. In this article we explore episodes in the history of infinitesimal calculus, in particular on Bonaventura Cavalieri (1598-1647), in order to highlight aesthetics aspects of mathematical demonstration. Initially, we present perspectives on aesthetics and mathematical demonstration in order to identify qualitative elements in the production of mathematical (formal) knowledge. Next, though a bibliographic research, we explore the use of notations/symbologies in the development of the Cavalieri's Principle and results involving the concept of integral. We emphasize aspects about language/notations and procedures in the enunciation and demonstration of some theorems/propositions of Cavalieri. We enphasize the notations o.l. (omnes linae), o.q. (omnes quadrata) e o.c. (omnes cubi), which means all lines, all the squares and all the cubes. The notion of omnes is related to the comtemporary concept of integral in Calculus. Finally, we mention nuances about how Leibniz (1646-1716), in creating a new symbology based on the notion of omnes linae, reorganized Cavalieri's aesthetics and developed new results on integrals.
\end{abstract}

Keywords: History of Mathematics. Infinitesimal Calculus. Mathematical Proof. 
Ricardo Scucuglia Rodrigues da Silva, Inocêncio Fernandes Balieiro Filho, Luis Augusto Schimidt Totti e Giovana Aparecida Bertolucci

Aspectos estéticos em demonstrações de Bonaventura Cavalieri

\section{Introdução}

Neste artigo exploramos, por meio de uma pesquisa bibliográfica, aspectos acerca de demonstrações de Bonaventura Cavalieri (1598-1647). Utilizamos perspectivas sobre estética para destacar notações utilizadas por Cavalieri.

De acordo com Baron (1985), existem muitos protagonistas na história do cálculo infinitesimal como Zenão de Eléia (489-430 a.C.), Arquimedes (287-212 a.C.), Euclides (300 a.C.), Fermat (1601-1665), Kepler (1571-1630), Galileu (1564-1642), Cavalieri (1598-1647), Pascal (1623-1662), Wallis (1616-1703), Descartes (15961650), Torricelli (1608-1647), Gregory (1638-1675), Barrow (1630-1677), Newton (1643-1727), Leibniz (1646-1716), Jakob Bernoulli (1654 - 1705), Johann Bernoulli (1667-1748), l’Hôpital (1661-1704), Berkeley (1685-1753), Maclaurin (1698-1746), Euler (1707-1783), Lagrange (1736-1813), d'Alambert (1717-1783), Cauchy (17891857), dentre outros. As publicações desses autores nos oferecem meios para identificarmos o papel fundamental da demonstração matemática (DM) na produção de conhecimentos matemáticos ao longo da história. Concomitantemente, reflexões sobre DM nos conduzem inevitavelmente a discussões da natureza filosófica, evocando conceitos como verdade e convencimento. De acordo com Silva (2002, p. 40, grifos do autor), uma DM tem diversificadas finalidades, dentre elas, "estabelecer a veracidade relativa de um enunciado" e "convencer-nos da veracidade da tese que demonstra". Portanto, DM nos remete a aspectos lógico-epistemológicos e retóricos.

A DM também suscita uma dimensão estética (PARTESON, 2013). Para realizar um estudo estético matemático, é importante conhecer a distinção entre fatos (ou produtos), que são as entidades produzidas ou encontradas na Matemática como teoremas, fórmulas, construções geométricas, e métodos (ou processos), que são ferramentas matemáticas, como demonstrações, heurísticas, algoritmos, estilos de notações, dentre outros. Os produtos são considerados mais análogos às obras de arte que os processos. Aliás, existem produtos matemáticos como triângulos, espirais e fractais que podem ser visualizados esteticamente sem uma estética matemática específica. Produtos que são entidades teóricas, carregados no sentido conceitual, como teoremas e equações, por exemplo, são, portanto, um desafio para a estética. Já a análise estética dos processos está bem integrada ao contexto matemático, devido ao fato de os métodos serem considerados ferramentas matemáticas. As demonstrações podem ser 
Ricardo Scucuglia Rodrigues da Silva, Inocêncio Fernandes Balieiro Filho, Luis Augusto Schimidt Totti e Giovana Aparecida Bertolucci

Aspectos estéticos em demonstrações de Bonaventura Cavalieri

consideradas processos com uma função geral e superior na Matemática. São ditas artefatos funcionais, ou seja, objetos humanamente criados, mas com funções epistêmicas na natureza, cuja interação se dá por meio da utilização. No entanto, é importante ter em mente que essa distinção nem sempre pode ser realizada com exatidão. A Teoria Matemática, por exemplo, não se encaixa inteiramente em produto ou processo, sendo tratada muitas vezes como uma beleza particular da Matemática. Assim como as teorias, os contraexemplos são objetos mistos, classificados ora como produtos, ora como processos (PATERSON, 2013).

\section{O Princípio de Cavalieri}

Bonaventura Cavalieri (1598-1647) estruturou sua obra intitulada Geometria indivisibilibus continuorum nova quadam ratione promota em sete partes (CAVALIERI, 1653) $^{5}$. Na Parte VII, por exemplo, Cavalieri enuncia um teorema atualmente conhecido como Princípio de Cavalieri (figura 1).

Figura 1 - Teorema de Cavalieri.

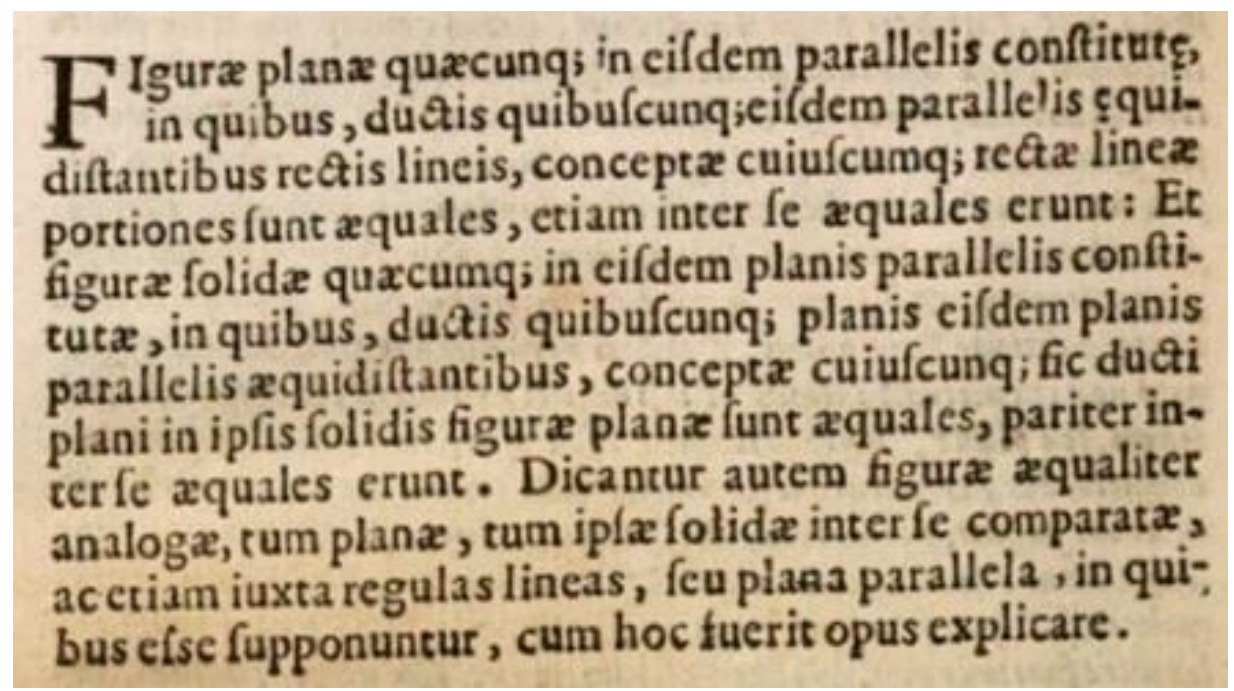

\footnotetext{
${ }^{5}$ De maneira geral, destacamos o seguinte acerca de Geometria: Cavalieri introduz na definição 1, livro II, o conceito fundamental (todas as retas de uma figura plana) de sua teoria; introduz na definição 2, livro II, o conceito fundamental (todos os planos ou coleção de todos os planos de uma figura sólida) de sua teoria; Cavalieri demonstra no teorema 1, livro II: As coleções de retas de figuras planas são grandezas que têm razão umas com as outras. Dois postulados. Cavalieri demonstra no teorema 2, livro II: Se duas figuras (plana ou sólida) são iguais, também são iguais suas coleções de retas (coleção de planos). Cavalieri demonstra no teorema 3, livro II: A razão entre duas figuras (plana ou sólida) é igual à razão entre suas coleções de retas, tomadas com respeito à regula. A Geometria compõem-se de sete livros; o livros 1 trata de geometria elementar de figuras planas e solidas; Cavalieri no livro 2 desenvolve o primeiro método de indivisíveis, o método coletivo e demostra alguns teoremas sobre coleções de indivisíveis; no livro 3, 4 e 5 aplica os teoremas anteriores para calcular áreas e volumes de figuras; no livro 6 calcula a área de espiral e volume de paraboloides; no livro 7 apresenta um novo método de indivisíveis, o método distributivo.
} 
Fonte: Cavalieri (1653).

Com base em Struik (1986), apresentamos a seguinte tradução do Teorema:

Se construirmos duas figuras planas quaisquer entre as mesmas paralelas e se ao traçarmos retas equidistantes as paralelas os segmentos que interceptam as figuras forem iguais, então as figuras planas serão também iguais e se construirmos duas figuras sólidas sobre os mesmos planos paralelos e ao traçarmos planos equidistantes dos planos paralelos as seções que interceptam as figuras forem iguais, então as figuras sólidas também serão iguais. (STRUIK, 1986 , p. 210, tradução nossa) ${ }^{6}$.

Uma maneira inicial de compreendermos esse enunciado no que diz respeito às figuras planas é a seguinte: sejam R e S regiões limitadas de um plano, e seja $\mathrm{r}$ uma reta desse plano. Suponha que, para toda reta s paralela a r, as interseções de R e S com s sejam vazias ou segmentos tais que a razão entre seus comprimentos é constante. Então, a razão entre as áreas de $\mathrm{R}$ e $\mathrm{S}$ é essa mesma constante. Podemos também enunciar esse Teorema da seguinte maneira: consideremos em um plano um sistema de coordenadas cartesianas $\mathrm{Oxy}$, e seja $\mathrm{R}$ a região delimitada por $\mathrm{y}=0, \mathrm{y}=\mathrm{b}>0$ e pelos gráficos das funções contínuas $x=f_{1}(y)$ e $x=f_{2}(y), 0 \leq y \leq b$, com $f_{1}(y) \leq f_{2}(y)$ para todo $y$. Seja $S$ a região delimitada por $\mathrm{y}=0, \mathrm{y}=\mathrm{b}$ e pelos gráficos das funções contínuas $\mathrm{x}=\mathrm{g}_{1}(\mathrm{y})$ e $\mathrm{x}=$ $\mathrm{g}_{2}(\mathrm{y}), 0 \leq \mathrm{y} \leq \mathrm{b}$, com $\mathrm{g}_{1}(\mathrm{y}) \leq \mathrm{g}_{2}(\mathrm{y})$ para todo $\mathrm{y}$. Suponhamos que exista $\mathrm{k}>0$ tal que $\mathrm{f}_{2}(\mathrm{y})-\mathrm{f}_{1}(\mathrm{y})=\mathrm{k}\left[\mathrm{g}_{2}(\mathrm{y})-\mathrm{g}_{1}(\mathrm{y})\right]$ para todo $\mathrm{y}$. Então $\mathrm{a}(\mathrm{R})=\mathrm{ka}(\mathrm{S})$. Em notação atual podemos demonstrar o seguinte:

$$
\begin{aligned}
& a(R)=\iint_{R} d x d y=\int_{0}^{h}\left[\int_{f_{1}(y)}^{f_{2}(y)} d x\right] d y=\int_{0}^{h}\left[f_{2}(y) \quad f_{1}(y)\right] d y= \\
& \int_{0}^{h} k\left[g_{2}(y) \quad g_{1}(y)\right] d y=k \int_{0}^{h}\left[g_{2}(y) \quad g_{1}(y)\right] d y=\ldots=k a(S) .
\end{aligned}
$$

Analogamente, podemos realizar uma abordagem com sólidos e as medidas dos volumes. Por um lado, de acordo com Baron (1985), o Princípio de Cavalieri é uma ideia importante na história do cálculo infinitesimal, pois diz respeito a um resultado fundamental ao pensamento diferencial e a noção de indivisíveis. Na realidade, a gênese desse problema nos remete a Demócrito (400 a.c.), que parece ter concebido a noção de

\footnotetext{
${ }^{6}$ The Theorem: If between the same parallels any two plane figures are constructed, and if in them, any straight lines being drawn equidistant from the parallels, the included portions of any one of these lines are equal, the plane figures are also equal to ne another; and if between the same parallel planes any solid figures are constructed, and if in them, any planes being drawn equidistant from the parallel planes, the included plane figures out of any one of the planes so drawn are equal, the solid figures likewise equal to one another. (STRUIK, 1986, p. 210).
} 
Ricardo Scucuglia Rodrigues da Silva, Inocêncio Fernandes Balieiro Filho, Luis Augusto Schimidt Totti e Giovana Aparecida Bertolucci

Aspectos estéticos em demonstrações de Bonaventura Cavalieri

sólido como sendo composto de seções planas paralelas a base; seções estas iguais ou diferentes. Considere, por exemplo, que um cone seja formado por seções (discos). No caso das seções serem iguais, não se trata de um cone, mas de um cilindro. No caso das seções serem diferentes, o cone será uma coleção de finos cilindros. Essa concepção de Demócrito foi utilizada por Arquimedes em $O$ Método e Cavalieri foi o primeiro a demonstrar, tendo um papel importante na história dos indivisíveis (BARON, 1985).

Por outro lado, a demonstração realizada por Cavalieri é consideravelmente complexa, seja do ponto de vista da linguagem/estrutura lógica, quanto do tipo de notações utilizadas (ou falta delas). Um dos principais aspectos que nos chama a atenção nesse enunciado de Cavalieri diz respeito à estética de sua linguagem. Baron (1985) comenta que as obras de Cavalieri se tornaram amplamente conhecidas, embora o uso dos conceitos de reta e superfícies indivisíveis possa ter gerado contradições. Para Baron (1985, p. 17), “a linguagem usada por Cavalieri para defender os seus métodos, usualmente calcada em termos de ideias derivadas de filósofos medievais, era em geral obscura e infeliz, não fazendo jus ao seu mérito". Nesta seção, optamos por apresentar o enunciado literal e uma demonstração simplificada utilizando notações atuais. Nas seções seguintes, abordaremos tanto as demonstrações de Cavalieri em sua linguagem original como também faremos abordagens utilizando notações contemporâneas.

Ainda, com base em Grattan-Guinness (2000), podemos destacar que a Geometria e a obra posterior de Cavalieri, Exercitationes geometricae sex ("Seis exercícios geométricos": 1647a), tornaram-se bem conhecidas entre os matemáticos, inspirando muitos a encontrar seus próprios métodos. Cavalieri apresentou dois métodos inspirado nos indivisíveis em Geometria, sendo denominados de métodos "coletivo" e "distributivo". Os seis primeiros dos sete livros da Geometria incorporam o método coletivo, e uma síntese dele é exposto no livro 1 de Exercitationes. A estrutura limitada desta seção não permite um relato completo do amplo espectro de conceitos e ideias que Cavalieri introduziu e desenvolveu nesses seis livros, mas o esboço a seguir fornece uma ideia de sua abordagem. 


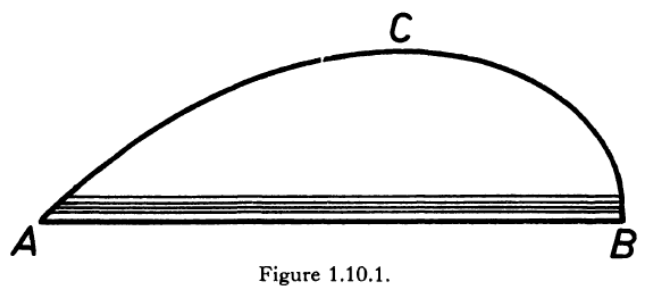

Seja dada uma figura plana $F=A B C$ limitada pela curva $A B C$, e uma reta $A B$, denominada "regula" (figura 1.10.1). Cavalieri imaginou que uma reta que, começando da posição da reta $A B$, desloca-se uniformemente paralela à reta $A B \mathrm{e}$ considerou o feixe de segmentos de reta paralelos que determinam a figura $F$ sobre a reta em suas posições durante o movimento. Ele nomeou esses segmentos de reta como "todas as retas da figura dada" ("omnes lineae propositae figurae"), e às vezes se referia a eles como "os indivisíveis da figura dada"; vamos denotá-los por $\mathcal{O}_{F}(l)$. Expresso em termos modernos, Cavalieri construiu uma função $F \rightarrow \mathcal{O}(l)$ (1.10.1) do conjunto de figuras planas em um conjunto que consiste em feixes de segmentos de reta paralelos. Ele então estendeu a teoria das grandezas de Eudoxo (ver o livro $\mathrm{V}$ de Os elementos de Euclides) para incluir suas novas grandezas $\left\{\mathcal{O}_{F}(l)\right\}$. Posteriormente ele estabeleceu - embora não de maneira satisfatória matematicamente - a relação fundamental $F_{1}: F_{2}=\mathcal{O}_{F_{1}}(l): \mathcal{O}_{F_{2}}(l)(1.10 .2)$ entre duas figuras planas (Cavalieri 1635a, Livro II, Teorema 3). Ao considerar a regula como um plano ele obteve de maneira semelhante a seguinte relação $S_{1}: S_{2}=$ $\mathcal{O}_{S_{1}}(p): \mathcal{O}_{S_{2}}(p)$ (1.10.3) em que $S_{i}$ é um sólido e $\mathcal{O}_{S_{i}}(p)$ são todos os planos pertencentes a esse sólido, para $i=1,2$. O objetivo de Cavalieri era encontrar a razão do lado esquerdo de (1.10.2) calculando a razão do lado direito. Ao fazê-lo, ele foi muito auxiliado por um postulado que conduz ao "teorema de Cavalieri" (descrito abaixo), com um uso habilidoso de resultados anteriores, os teoremas sobre figuras semelhantes e o conceito de potências de segmentos de reta. $\mathrm{O}$ postulado (1635a, Corolário do Teorema 4 do Livro II) afirma que, se em duas figuras $F_{1}$ e $F_{2}$ com a mesma altura, cada par de segmentos de reta correspondentes (isto é, segmentos de reta a distâncias iguais da regra comum) tem a mesma razão, então $\mathcal{O}_{F_{1}}(l)$ e $\mathcal{O}_{F_{2}}(l)$ também têm essa mesma razão. Em notação moderna e usando a figura 1.10.2, se para todo $x$ tal que $0<x<a$ tem-se $f_{1}(x): f_{2}(x)=$ $b: c, \quad$ então, $\quad \mathcal{O}_{F_{1}}(l): \mathcal{O}_{F_{2}}(l)=b: c$. (1.10.4). Isto, junto com (1.10.2), estabelece imediatamente o "teorema de Cavalieri": se para todo $x$ tal que $0<x<a$ tem-se $f_{1}(x): f_{2}(x)=b: c$, então $F_{1}: F_{2}=b: c(1.10 .5)$ (1635a, Livro II, Teorema 4).

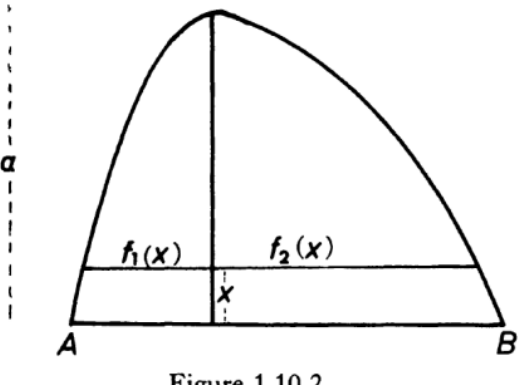

Figure 1.10.2.

(GRATTAN-GUINNESS, 2000, p. 33-34, tradução nossa). ${ }^{7}$

\footnotetext{
${ }^{7}$ A referência ao texto de 1635 de Cavaliari, diz respeito a obra "Geometria indivisibilibus continuorum nova quadam ratione promota". No escopo geral deste artigo, utilizamos a edição publicada em 1653 dessa obra de Cavalieri.
} 
A abordagem proposta por Grattan-Guinness (2000) nos oferece aspectos relevantes para entendermos em notação atual abordagens conduzidas por Cavalieri. Dentre esses aspectos, destacamos a noção de omnes, que pode ser entendida como a soma de todas. Na próxima seção faremos esse tratamento, já abordando Proposições em textos originais de Cavalieri.

\section{Integração em Cavalieri}

Além do Princípio de Cavalieri, Struik (1986) destaca um conjunto de proposições apresentadas por Cavalieri (1647) na Parte IV do livro intitulado Exercitationes geometricae sex, o qual diz respeito a um método de integração. De acordo com Struik (1986), esse método consistia em comparar os indivisíveis entre duas figuras, sendo este considerado um "procedimento perigoso", pois não era claro como e quais indivisíveis deveriam ser comparados. Identificamos, portanto, uma ressalva de natureza estética/procedimental apontada por Struik (1986) sobre essa abordagem de Cavalieri em termos de DM. No entanto, a abordagem de Cavalieri nos chama atenção do ponto de vista da notação utilizada, visto que nesse método Cavalieri adicionou esses indivisíveis usando expressões como omnes linae (o.1.) = all lines (a.1.), que significa todas as retas ou soma de todas as retas. Dessa maneira, Cavalieri explorou um importante resultado do cálculo, o qual podemos enunciar com notação atual da seguinte maneira, sendo $\mathrm{n}$ inteiro positivo:

$$
{ }_{0}^{a} x^{n} d x=\frac{a^{n+1}}{n+1}
$$

Na Parte IV do livro de Cavalieri (1647, p. 273, tradução nossa), é enunciado o seguinte: "Proposição XXI. Na mesma figura a mesma regra: todos os cubos do paralelogramo AD serão o quádruplo de todos os cubos de cada um dos ditos triângulos ACF, FDC"8. A demonstração original desta proposição é a seguinte (figura 2):

Figura 2 - Proposição XXI - Cavalieri (1647) - Livro IV.

\footnotetext{
${ }^{8}$ Propositio XXI. In codem schemate, regula cadem: omnes cubi paralelogramms, AD, quadrupli crunt omnium cuborum cuiusvis diforum triangulorum, ACF, FCD.
} 


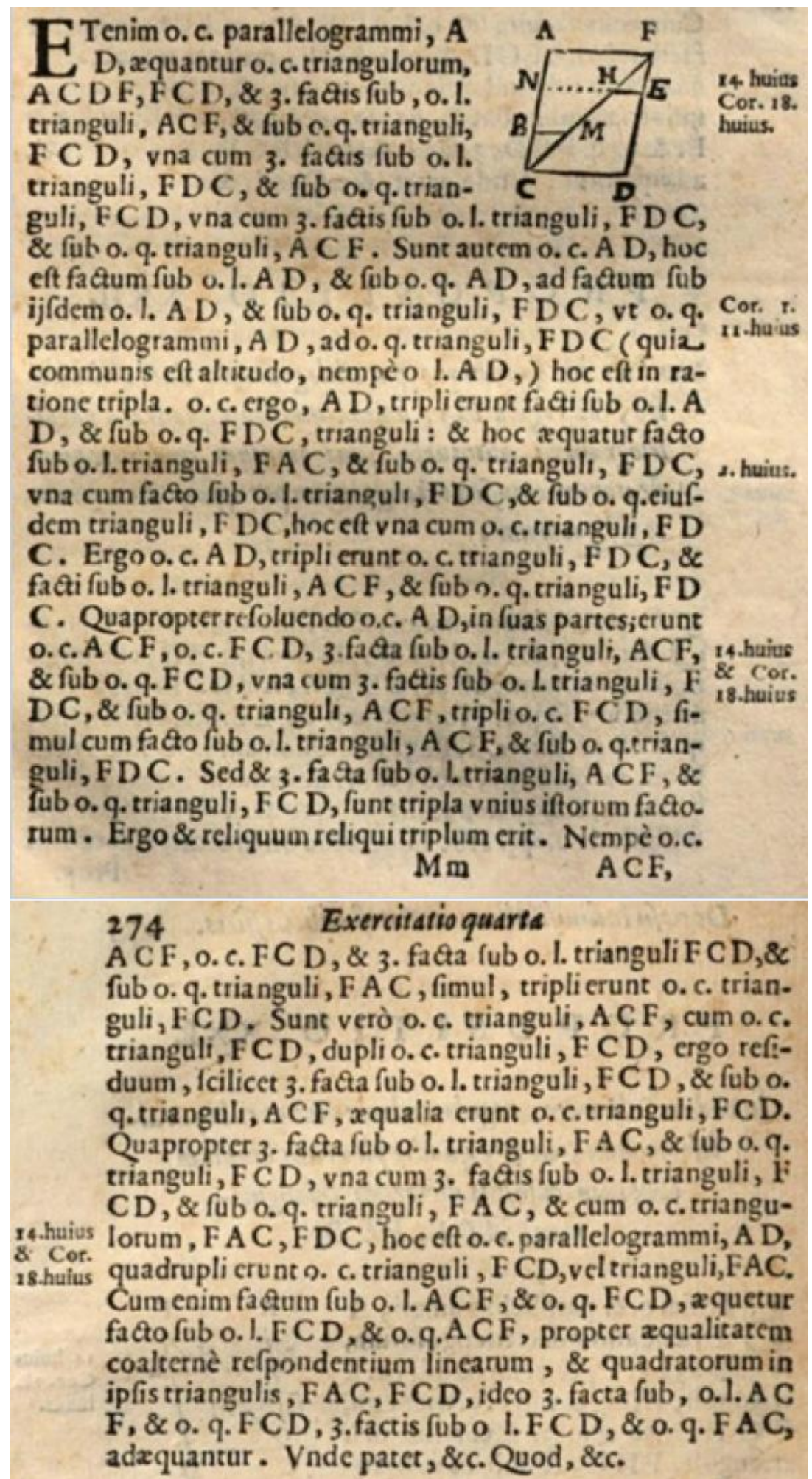

Fonte: Cavalieri (1647).

Nossa tradução, tendo como apoio Struik (1986), é a seguinte: 
Agora a.c. dos AD são o produto de a.l. $\mathrm{AD}$ por a.s. $\mathrm{AD}$, e este está para o produto de a.l. de $\mathrm{AD}$ por a.s. do triângulo FDC, assim como a.s. do paralelogramo AD está para a.s. do triângulo FDC (pois possuem alturas iguais, a saber a.l. AD), e esta razão é 3. Então, a.c. de $\mathrm{AD}$ são iguais a três vezes o produto de a.l. de $\mathrm{AD}$ por a.s. do triângulo FDC, e este é igual ao produto de a.l. do triângulo ACF por a.s. do triângulo FDC

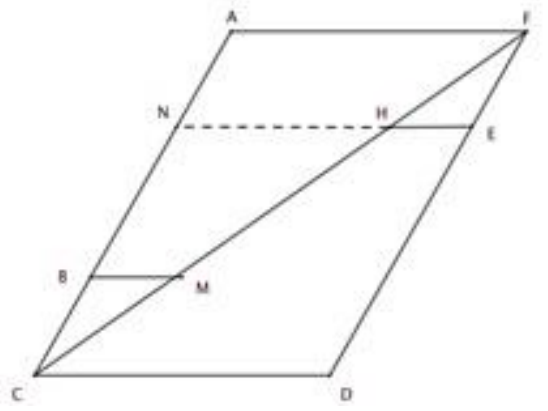
mais o produto de a.l. do triângulo FDC por a.s. do mesmo triângulo FDC, e este é igual a a.c. do triângulo FDC. Então a.c. de AD será três vezes a soma de a.c. do triângulo FDC e o produto de a.l do triângulo ACD por a.s. do triângulo FDC. Se agora decompormos a.c. de $\mathrm{AD}$ em suas partes, então obteremos a.c. de ACF mais a.c. de FDC mais três vezes o produto de a.l. do triângulo FDC por a.s. do triângulo ACF mais três vezes o produto de a.l. do triângulo ACF por a.s. do triângulo FDC. Mas três vezes o produto de a.l. do triângulo ACF por a.s. do triângulo FDC é igual a três vezes o mesmo produto. Se o descartarmos então do que descartamos, ainda permanece. Logo a.c. de ACF mais a.c. de FDC é igual a três vezes o produto de a.l. do triângulo FDC por a.s. do triângulo FAC são iguais a três vezes a.c. do triângulo FDC. Agora a.c. do triângulo ACF mais a.c. do triângulo FDC são iguais a duas vezes a.c. do triangulo FDC desde que a.c. do triângulo ACF será igual a a.c. do triângulo FCD. Então três vezes o produto de a.l. do triângulo ACF por a.s. do triângulo FCD junto com três vezes o produto de a.l. do triângulo FCD por a.l. do triângulo FAC e a.c. dos triângulos $\mathrm{ACF}$, FDC, que é a.c. do paralelogramo AD, são iguais ao quádruplo de a.c. do triângulo FDC (ou triângulo FAC). Isto é assim porque o produto de a.l. de FDC por a.s. de FDC é igual ao produto de a.l. de FDC por a.s. de ACF, e isto é assim por causa da igualdade de retas e seus quadrados nos triângulos FDC, ACF. Assim três vezes o produto de a.l. de ACF por a.s. de FDC são iguais a três vezes o produto de FDC por a.s. de ACF. Isto torna clara a demonstração (CAVALIERI, 1647, p. 274, tradução nossa).

Consideramos, de um ponto de vista estético, o entendimento da demonstração da Proposição XXI de Cavalieri (1647) de difícil compreensão, devido a sua linguagem e maneira de uso de notações. Notamos que, do ponto de vista estético, Cavalieri não utilizou notações algébricas. O primeiro passo para compreender a demonstração de Cavalieri, provavelmente seja identificar o significado das expressões a.1., a.s. e a.c. que, respectivamente, podem ser traduzidos como todas as retas, todos os quadrados e todos os cubos (dos triângulos ou do paralelogramo). Além disso, a demonstração envolve conhecimentos prévios sobre expansão binomial em potencia inteiras.

Para compreendermos o raciocínio da demonstração da Proposição XXI, de acordo com Struik (1986), vamos primeiramente, utilizando notação atual, mostrar que a área do paralelogramo AFCD mede o dobro da área do triângulo AFC, obtido pela diagonal FC. Pela figura na demonstração, temos que NE // BM // AF, de modo que HE 
Ricardo Scucuglia Rodrigues da Silva, Inocêncio Fernandes Balieiro Filho, Luis Augusto Schimidt Totti e Giovana Aparecida Bertolucci

Aspectos estéticos em demonstrações de Bonaventura Cavalieri

$=\mathrm{BM}$. Consideremos $\mathrm{HE}=\mathrm{x}, \mathrm{NH}=\mathrm{y}$ e $\mathrm{AF}=\mathrm{a}$. Então, $\mathrm{x}+\mathrm{y}=\mathrm{a}$. Consequentemente, $\Sigma \mathrm{x}+\Sigma \mathrm{y}=\Sigma$ a quando nós somamos todas as linhas paralelas NE de AF a CD. Para todo $\mathrm{NH}$ existe apenas um (CD - BM) correspondente. Mas como $\mathrm{HE}=\mathrm{BM}, \Sigma \mathrm{x}=\Sigma \mathrm{y}$. Consequentemente, $\Sigma \mathrm{x}=1 / 2 \Sigma \mathrm{a}$. Assim, $\Sigma \mathrm{x}$ é a área do triângulo $\mathrm{FCD}, \Sigma \mathrm{a}$ do paralelogramo. Se fossemos introduzir o símbolo $\Delta \mathrm{x}$, poderíamos escrever $(\mathrm{x}+\mathrm{y}) \Delta \mathrm{x}=\mathrm{a}$ $\Delta \mathrm{x}$, e assim obteríamos $\Sigma \mathrm{x} \Delta \mathrm{x}=1 / 2 \sum \mathrm{a} \Delta \mathrm{x}=1 / 2$ a $\sum \mathrm{x}=1 / 2$ a, o que mostra que a fórmula de Cavalieri é equivalente a ${ }_{0}^{a} x^{n} d x=\frac{1}{2} a^{2}$

De fato, temos o seguinte a partir de $(x+y)^{2}=a^{2}$.

$$
\begin{gathered}
\Sigma \mathrm{x}^{2}+2 \Sigma \mathrm{xy}+\Sigma \mathrm{y}^{2}=\Sigma \mathrm{a}^{2} \\
2 \Sigma \mathrm{x}^{2}+2 \Sigma \mathrm{xy}=\Sigma \mathrm{a}^{2} .
\end{gathered}
$$

Para encontrarmos $\Sigma x y$, nós podemos escrever $x=1 / 2 a+z, y=1 / 2 a-z$. Então:

$$
\Sigma x y=\Sigma\left(1 / 4 \mathrm{a}^{2}-\mathrm{z}^{2}\right)=1 / 4 \Sigma \mathrm{a}^{2}-\Sigma \mathrm{z}^{2} .
$$

De triângulos semelhantes nós determinamos $\Sigma \mathrm{z}^{2}=1 / 4 \Sigma \mathrm{x}^{2}$. Consequentemente,

$$
2 \Sigma \mathrm{x}^{2}+1 / 2 \sum \mathrm{a}^{2}-1 / 2 \sum \mathrm{x}^{2}=\Sigma \mathrm{a}^{2} \text { ou } \Sigma \mathrm{x}^{2}=1 / 3 \sum \mathrm{a}^{2} .
$$

Tendo realizado tais considerações, abordemos agora a Proposição XXI em notação moderna. Consideremos $\mathrm{AF}=\mathrm{CD}=\mathrm{a}, \mathrm{HE}=\mathrm{BM}=\mathrm{x}, \mathrm{NH}=\mathrm{y}, \mathrm{x}+\mathrm{y}=\mathrm{a}$. Assim, a Proposição XXI enuncia que $\Sigma \mathrm{a}^{3}=4 \Sigma \mathrm{x}^{3}=4 \Sigma \mathrm{y}^{3}$. O raciocínio que segue é o seguinte:

$$
\begin{gathered}
\Sigma(\mathrm{x}+\mathrm{y})^{3}=\Sigma \mathrm{x}^{3}+\Sigma \mathrm{y}^{3}+3 \Sigma \mathrm{xy}^{2}+3 \Sigma \mathrm{x}^{2} \mathrm{y} . \text { Então: } \\
\Sigma(\mathrm{x}+\mathrm{y})^{3}: \Sigma(\mathrm{x}+\mathrm{y}) \mathrm{x}^{2}=\Sigma(\mathrm{x}+\mathrm{y})^{2}: \Sigma \mathrm{x}^{2}=3: 1 \\
\Sigma(\mathrm{x}+\mathrm{y})^{3}=3 \Sigma(\mathrm{x}+\mathrm{y}) \mathrm{x}^{2} \\
\Sigma(\mathrm{x}+\mathrm{y}) \mathrm{x}^{2}=\Sigma \mathrm{x}^{2} \mathrm{y}+\Sigma \mathrm{x}^{2} \mathrm{x}=\Sigma \mathrm{x}^{2} \mathrm{y}+\Sigma \mathrm{x}^{3} \\
\Sigma(\mathrm{x}+\mathrm{y})^{3}=3 \Sigma \mathrm{x}^{3}+3 \Sigma \mathrm{x}^{2} \mathrm{y} \\
\Sigma(\mathrm{x}+\mathrm{y})^{3}=\Sigma \mathrm{x}^{3}+\Sigma \mathrm{y}^{3}+3 \Sigma \mathrm{xy}^{2}+3 \Sigma \mathrm{x}^{2} \mathrm{y} .
\end{gathered}
$$

Subtraindo $3 \Sigma \mathrm{x}^{2} \mathrm{y}$ de ambos os membros da expressão, temos:

$$
\Sigma \mathrm{x}^{3}+\Sigma \mathrm{y}^{3}+3 \Sigma \mathrm{xy}^{2}=3 \Sigma \mathrm{x}^{3}
$$

Mas como $\Sigma \mathrm{x}^{3}+\Sigma \mathrm{y}^{3}=2 \Sigma \mathrm{x}^{3}, \Sigma \mathrm{x}^{3}=\Sigma \mathrm{y}^{3}$,

Nós encontramos $3 \sum \mathrm{x}^{2} \mathrm{y}=3 \Sigma \mathrm{x}^{2} \mathrm{y}=\Sigma \mathrm{x}^{3}$.

Consequentemente, $\Sigma \mathrm{x}^{3}+\Sigma \mathrm{y}^{3}+3 \Sigma \mathrm{xy}^{2}+3 \Sigma \mathrm{x}^{2} \mathrm{y}=\Sigma(\mathrm{x}+\mathrm{y})^{3}=4 \Sigma \mathrm{x}^{3}=4 \Sigma \mathrm{y}^{3} \bullet$ 
Ricardo Scucuglia Rodrigues da Silva, Inocêncio Fernandes Balieiro Filho, Luis Augusto Schimidt Totti e Giovana Aparecida Bertolucci

Aspectos estéticos em demonstrações de Bonaventura Cavalieri

Esse raciocínio pode ser sumarizado da seguinte maneira (STRUIK, 1969): Com o objetivo de encontrar $\Sigma \mathrm{x}^{3}$, escrevemos $\Sigma(\mathrm{x}+\mathrm{y})^{3}=2 \Sigma \mathrm{x}^{3}+6 \Sigma \mathrm{x}^{2} \mathrm{y}=\Sigma \mathrm{a}^{3}$. Mas $\Sigma \mathrm{a}^{3}=\mathrm{a}$ $\Sigma \mathrm{a}^{2}=\mathrm{a}\left(2 \Sigma \mathrm{x}^{2}+2 \Sigma \mathrm{xy}\right)=2 / 3 \Sigma \mathrm{a}^{3}+2 \mathrm{a} \Sigma \mathrm{xy}=2 / 3 \Sigma \mathrm{a}^{3}+2 \Sigma(\mathrm{x}+\mathrm{y}) \mathrm{xy}=2 / 3 \Sigma \mathrm{a}^{3}+4 \Sigma \mathrm{x}^{2} \mathrm{y}$. Assim, $\Sigma \mathrm{x}^{2} \mathrm{y}=1 / 12 \Sigma \mathrm{a}^{3}$ e $\Sigma \mathrm{x}^{3}=1 / 4 \Sigma \mathrm{a}^{3}$. De maneira similar, podemos desenvolver $\mathrm{x}^{\mathrm{n}} \mathrm{a}$ partir de $\mathrm{x}^{3}, \mathrm{n}>3$, e provar que $\Sigma \mathrm{x}^{\mathrm{n}}=[1 /(\mathrm{n}+1)] \mathrm{a}^{\mathrm{n}}$, o que é equivalente a integral ${ }_{0}^{a} x^{n} d x=\frac{a^{n+1}}{n+1}$.

Utilizando essa notação, dizemos que a Proposição XXII de Cavalieri enuncia o seguinte: Prove que $5 \Sigma \mathrm{x}^{4}=\Sigma \mathrm{a}^{4}$.

Agora, daremos atenção à Proposição XXIII (figura 3):

Figura 3 - Proposição XXIII- Cavalieri (1647) - Livro IV.

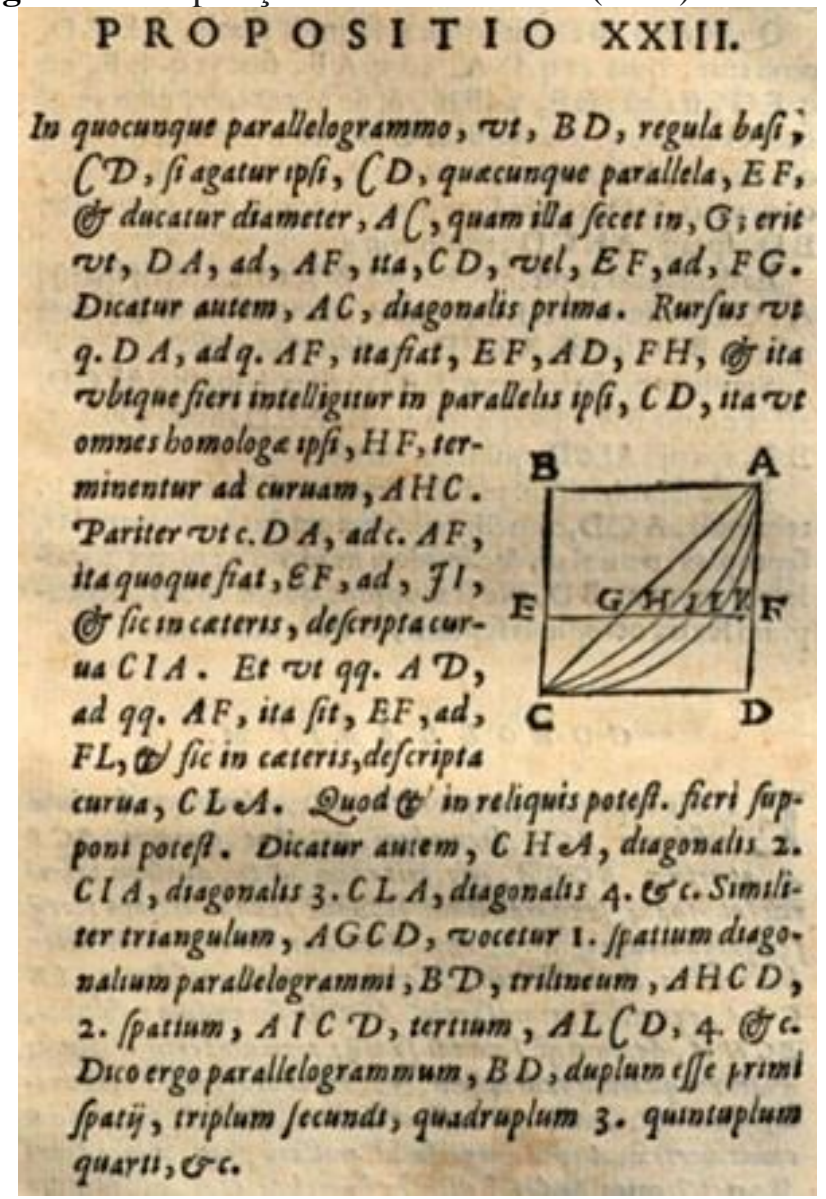

Fonte: Cavalieri (1647).

Proposição XXIII. Em qualquer paralelogramo, tal como $\mathrm{BD}$ com base $\mathrm{CD}$, tracemos uma paralela arbitrária $\mathrm{EF}$ a $\mathrm{CD}$ e a diagonal $\mathrm{AC}$, interceptando $\mathrm{EF}$ em G. Então DA:AF = CD ou EF:FG. AC é a primeira

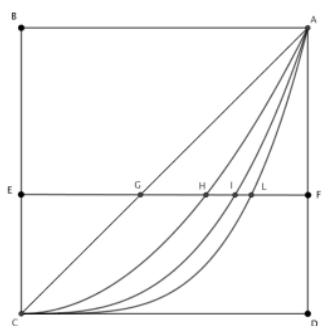


diagonal. Depois, seja $\mathrm{H}$ o ponto sobre $\mathrm{EF}$ tal que $\mathrm{AD}^{2}: \mathrm{AF}^{2}=\mathrm{EF}: \mathrm{FH}$, e assim em todas as paralelas a $\mathrm{CD}$, de tal forma que todas as retas como essa, HF, terminem numa curva CHA. Do mesmo modo construímos uma curva CIA, onde $\mathrm{DA}^{3}: \mathrm{AF}^{3}=\mathrm{EF}: \mathrm{FI}$, uma curva CLA tal que $\mathrm{DA}^{4}: \mathrm{AF}^{4}=\mathrm{EF}: \mathrm{FL}$ etc. CHA é a segunda diagonal, CIA é a terceira, CLA é a quarta, etc. E do mesmo modo AGCD é a primeira diagonal espacial do paralelogramo BD, a figura AHCD é a segunda, AICD é a terceira, ALCD é a quarta, etc. Então eu digo que o paralelogramo BD é duas vezes o primeiro, três vezes o segundo, quatro vezes o terceiro, cinco vezes o quarto espaço, etc. (CAVALIERI, 1647, p. 279, tradução nossa). ${ }^{9}$

Na figura 4, apresentamos a demonstração da Proposição XXIII:

Figura 4 - Demonstração da Proposição XXIII.

Vod enim parallelogrammum, B D, duplum fit I.
fpatij, A CD, patet quia per Prop.19. o. I. BD,
dupla

Fonte: Cavalieri (1647).

Segue nossa tradução:

Dessa forma fica evidente que o paralelogramo BD é o dobro do (primeiro) espaço ACD, porque pela Prop. 19, a.l. de BD são o dobro de a.l. de ACD, e assim, pela Prop. 3 do Segundo Livro da Geom. Ind., BD é o dobro do espaço $\mathrm{ACD}$. E de fato está provado que BD é o triplo do segundo espaço ALCD, pois

\footnotetext{
${ }^{9}$ Tradução nossa fundamentada em Struik (1986).
} 
assim como $\mathrm{DA}^{2}$ está para $\mathrm{AF}^{2}$, ou assim como $\mathrm{EF}^{2}$ está para $\mathrm{FG}^{2}$, também $\mathrm{EF}$ está para FH; e da mesma forma para os demais, daí, tal como a.s. de BD estão para a.s. do triângulo ACD, também a.l. de BD estão para a.l. do espaço AHCD, e, como resultado portanto, assim como BD está para AHCD, então a.s. de BD são o triplo de a.s. do triângulo $\mathrm{ACD}$ e, deste modo, BD será o triplo do espaço AHCD. Demonstraremos, pela mesma razão, que, assim como a.l. de BD estão para a.l. do terceiro espaço AICD, também a.c. de BD estão para a.c. do triângulo $\mathrm{ACD}$, ou seja, igualmente $\mathrm{BD}$ é o quádruplo do espaço $\mathrm{AICD}$. De modo semelhante, provaremos que a.l. de BD estão para a.l. do espaço ALCD assim como a.qq. de BD estão para a.qq. do triângulo $\mathrm{ACD}$, certamente do mesmo modo que BD é o quíntuplo do espaço ALCD. E assim, provamos que a.s. de BD, sucessivamente subsequentes, são o sêxtuplo, o sétuplo, o óctuplo, etc. de a.s. do triângulo ACD em relação aos de mesmo grau. Do mesmo modo, demonstra-se que o paralelogramo BD é o sêxtuplo do quinto espaço, o sétuplo do sexto, o óctuplo do sétimo, e assim até o infinito (CAVALIERI, 1647, p 279280, tradução nossa).

Para compreendermos o raciocínio de Cavalieri, utilizamos notação atual como sugere Struik (1986). Seja $\mathrm{CE}=\mathrm{a}, \mathrm{DA}=\mathrm{b}, \mathrm{AF}=\mathrm{z}, \mathrm{FG}=\mathrm{x}, \mathrm{FH}=\mathrm{x}_{1}, \mathrm{FI}=\mathrm{x}_{2}, \mathrm{FL}=\mathrm{x}_{3}$, $\mathrm{GE}=\mathrm{y}, \mathrm{HE}=\mathrm{y}_{1}$ e a área do paralelogramo $\mathrm{BD}=\Sigma \mathrm{a}=\mathrm{P}$. Então a demonstração é a seguinte: O paralelogramo BD é o dobro de $\mathrm{ACD}, \mathrm{P}=\Sigma \mathrm{a}=2 \Sigma \mathrm{x}$, como demonstrado em proposição anterior. Como $\mathrm{b}^{2}: \mathrm{z}^{2}=\mathrm{a}^{2}: \mathrm{x}^{2}=\mathrm{a}: \mathrm{x}_{1}$, temos $\mathrm{a}^{2}: \sum \mathrm{x}^{2}=\mathrm{a}: \sum \mathrm{x}_{1}$ e, de acordo com proposição anterior, $\Sigma \mathrm{x}^{2}=1 / 3 \Sigma \mathrm{a}^{2}=1 / 3$ aP. Assim, concluímos que $\mathrm{a}^{2}: 1 / 3$ $\mathrm{aP}=\mathrm{a}: \Sigma \mathrm{x}_{1}$ ou $\mathrm{P}=3 \Sigma \mathrm{x}_{1}$. Em outras palavras, o paralelogramo BD é o triplo do espaço AHCD. Da mesma maneira podemos mostrar que $\mathrm{a}^{3}: \mathrm{x}^{3}=\mathrm{a}: \mathrm{x}^{2}$ e $\sum \mathrm{x}^{3}=1 / 4 \sum \mathrm{a}^{3}=1 / 2 \mathrm{a}^{2} \mathrm{P}$ que $\mathrm{P}=4 \Sigma \mathrm{x}^{2}$, ou o paralelogramo BD é o quadruplo de AICD, e assim sucessivamente.

Portanto, de um ponto de vista estético, abordamos entendimentos sobre a linguagem e notações utilizadas por Cavalieri, perpassando sobre elementos da estrutura de suas demonstrações. Além da grafia e usufruto do latim, com referências ao uso de imagens, enfatizamos o uso das notações referente ao conceito de omnes, isto é, omnes linae (o.l), omnes quadrata (o.q.) e omnes cubi (o.c.). Esse conceito está relacionado à notação $\Sigma$ utilizada por Struik (1986), que por sua vez, no Cálculo comtemporâneo, possui relação com o conceito de integração definida.

\section{Considerações Finais}

Nosso objetivo neste artigo foi, por meio de uma pesquisa bibliográfica de natureza qualitativa, explicitar aspectos estéticos em demonstrações de Bonaventura Cavalieri em episódios importantes na História do Cálculo Infinitesimal no que se refere 
Ricardo Scucuglia Rodrigues da Silva, Inocêncio Fernandes Balieiro Filho, Luis Augusto Schimidt Totti e Giovana Aparecida Bertolucci

Aspectos estéticos em demonstrações de Bonaventura Cavalieri

à noção de indivisíveis. Com base em texto original em latim (CAVALIERI, 1647), apresentamos a linguagem original de Cavalieri em algumas Proposições, as quais se caracterizam pela ausência de notações algébricas e difícil compreensão. Com base em referências como Struik (1986), buscamos esclarecer seus enunciados e demonstrações por meio de traduções e utilização de notações atuais. De um ponto de vista estético, exploramos produtos e processos de um fazer matemático (PARTESON, 2013). Nesse sentido, cabe destacar que as abordagens de Cavalieri foram fundamentais para o desenvolvimento do Cálculo Infinitesimal por Leibniz. $\mathrm{Na}$ realidade, Leibniz desenvolveu diversos de seus resultados por ter simplificado a notação de Cavalieri. Para representar omnes linae $y$, Leibniz introduziu um S estilizado da seguinte maneira: $\int y d x$. Trata-se, tanto de um ponto de vista conceitual como estético, de um episódio relevante da História do Cálculo Infinitesimal.

\section{Referências}

BARON, M. E. Curso de História da Matemática: origens e desenvolvimento do Cálculo - Unidade 2: os indivisíveis. Brasília: Editora Universidade de Brasília, 1985.

CAVALIERI, B. Exercitationes geometricae sex. Bologna: Typis Jacobi Montij, 1647.

CAVALIERI, B. Geometria indivisibilibus continuorum nova quadam ratione promota. Bologna: Typographia de Ducijs, 1653.

GRATTAN-GUINNESS, I. From the calculus to set theory, 1630-1910. An Introductory History. New Jersey: Princeton University Press, 2000.

PARTESON, G. D. The aesthetics of mathematical proofs (Masters Thesis of Arts). Edmonton, Alberta, Canada: University of Alberta, 2013.

SILVA, J. J. A demonstração matemática na perspectiva da lógica matemática. Boletim de Educação Matemática. v. 15, n. 8, p. 68-78, 2002.

STRUIK, D. J. A source book in mathematics, 1200-1800. Princeton, NJ: Princeton University Press, 1986.

Recebido em: 17 / 09 / 2020

Aprovado em: 27 / 12 / 2020 